\section{ORIGINAL RESEARCH}

N. Akutsu

K. Hosoda

A. Fujita

E. Kohmura

\title{
A Preliminary Prediction Model with MR Plaque Imaging to Estimate Risk for New Ischemic Brain Lesions on Diffusion-Weighted Imaging after Endarterectomy or Stenting in Patients with Carotid Stenosis
}

\begin{abstract}
BACKGROUND AND PURPOSE: Findings on MR imaging of carotid plaques correlate with histologic findings and may be useful in identifying vulnerable plaques. The objective of this study was to show how MR imaging findings and clinical factors could be used to construct a preliminary model and a nomogram for predicting the risk of new ischemic lesions on DWI following CEA or CAS.
\end{abstract}

MATERIALS AND METHODS: One hundred four patients with carotid stenosis undergoing treatment (63 CEA, 41 CAS) were prospectively enrolled (mean age, $71.7 \pm 7.0$ years; 11 women). T1-SIR and T2-SIR of carotid plaque were measured on MR imaging. Associations among carotid MR imaging findings, treatment procedures, degree of stenosis, cardiovascular risk factors, and occurrence of new ischemic lesions on DWI 1 day after treatment were studied by multivariate logistic regression.

RESULTS: One stroke occurred after CAS (2.4\%), and none after CEA. New DWI lesions after treatment were observed in 25 patients $(24 \%)$. Our preliminary prediction model demonstrated that T1-SIR (OR [per 0.5 increase], 3.99; 95\% Cl, 2.18-7.31; $P<.0001$ ) and CAS (OR, 2.06; 95\% Cl, $1.01-4.24 ; P=.048$ compared with CEA) were positively associated with new DWI lesions on posttreatment DWI scans. T2-SIR (OR [per 0.5 increase], $0.74 ; 95 \% \mathrm{Cl}, 0.55-0.98 ; P=.037$ ) was negatively associated. The $\mathrm{C}$-index of this model was 0.79 (95\% $\mathrm{Cl}, 0.69-0.89)$, which indicated some utility in predicting the response.

CONCLUSIONS: Our preliminary prediction model and nomogram may provide an individualized risk estimate of new ischemic lesions after CEA or CAS and useful information for decision-making regarding treatment strategy.

ABBREVIATIONS: $\mathrm{BB}=$ black-blood; $\mathrm{CAS}=$ carotid artery stenting; $\mathrm{CEA}=$ carotid endarterectomy; $\mathrm{Cl}=$ confidence interval; $\mathrm{DM}=$ diabetes mellitus; $\mathrm{ETL}=$ echo-train length; $\mathrm{FA}=$ flip angle; $\mathrm{IHD}=$ ischemic heart disease; IR = inversion recovery; MPRAGE = magnetization-prepared rapid acquisition of gradient echo; OR = odds ratio; SIR = signal-intensity ratio; SPIR = spectral presaturation with inversion recovery

$\mathbf{T}^{\mathrm{h}}$ hromboembolism from carotid plaque is 1 of the major causes of ischemic stroke. Several randomized controlled trials have demonstrated the prophylactic effectiveness of CEA in patients with high-grade carotid stenosis. ${ }^{1,2}$ CAS has recently emerged as an alternative to CEA for the treatment of carotid stenosis. ${ }^{3}$ Although CAS is a less invasive procedure, one of its disadvantages is the considerably high incidence of distal emboli, though most are subclinical. ${ }^{4}$ Indications for CEA and CAS have been based on the degree of carotid stenosis and symptoms.

Recent histopathologic studies, however, have demonstrated increasing evidence of the existence of "vulnerable plaque," which is characterized by the presence of a lipid-rich necrotic core, ruptured fibrous cap, and intraplaque hemorrhage, each of which is associated with an increased risk for

Received September 15, 2011; accepted after revision December 2.

From the Department of Neurosurgery, Kobe University Graduate School of Medicine, Kobe, Japan.

Please address correspondence to Kohkichi Hosoda, MD, Department of Neurosurgery, Kobe University Graduate School of Medicine, 7-5-1, Kusunoki-cho, Chuo-ku, Kobe, 6500017, Japan; e-mail: khosoda@med.kobe-u.ac.jp

http://dx.doi.org/10.3174/ajnr.A3002 thromboembolic events resulting in ischemia. ${ }^{5,6}$ Therefore, alternative criteria to identify high-risk plaque features may improve risk assessment and allow targeted intervention.

Findings on MR imaging of carotid plaques have been reported to correlate with histologic findings in identifying the principal components of carotid lesions, such as the lipid-rich necrotic core ${ }^{7-9}$ intraplaque hemorrhage, ${ }^{7,9-12}$ and ruptured fibrous cap. ${ }^{13}$ Some studies have demonstrated that the presence of vulnerable features, such as intraplaque hemorrhage, which was depicted as hyperintense signal intensity by T1weighted carotid MR imaging, ${ }^{14,15}$ was associated with an increased risk of subsequent ischemic events on clinical followup. ${ }^{16,17}$ Acute intraplaque hemorrhage was histologically characterized by intact red blood cells with intracellular methemoglobin and appeared hyperintense on T1-weighted images and iso- to hypointense on T2-weighted images. ${ }^{10,18}$ Most recent studies have demonstrated that intraplaque hemorrhage as detected by preoperative carotid MR imaging could predict particulate embolization during the dissection phase of CEA ${ }^{19}$ or after the treatment procedure. ${ }^{20,21}$ Therefore, MR imaging of carotid plaque, especially T1- and T2-weighted images, may permit the development of a risk-prediction algorithm. Prediction models may be graphically depicted as 
nomograms, which are statistically based tools that provide overall probability of a specific outcome for an individual patient.

We designed a prospective cohort study to investigate the association between plaque findings on T1- and T2-weighted MR imaging and the risk of new ischemic lesions on posttreatment MR imaging and to investigate the associations with cardiovascular risk factors and treatment options (ie, CEA and CAS). The goal of this study was to construct a preliminary clinical prediction model and nomogram with findings of carotid plaque MR imaging and clinical risk factors to estimate the risk for new ischemic lesions on MR imaging after CEA or CAS.

\section{Materials and Methods}

Patients considered for CEA or CAS were prospectively recruited between August 2006 and February 2011 in our hospital. The inclusion criteria of the current study were carotid stenosis of $\geq 50 \%$ for symptomatic cases and $\geq 60 \%$ stenosis for asymptomatic cases according to the criteria of NASCET and the Asymptomatic Carotid Atherosclerosis Study. ${ }^{1,2}$ Symptomatic patients were defined as those who had experienced amaurosis fugax, a transient ischemic attack, or a stroke in the territory of the ipsilateral carotid artery within 6 months before entry. Exclusion criteria were emergency CEA or CAS for stroke in evolution or crescendo transient ischemic attack, major disabling stroke, contraindications for MR imaging, or patients in whom waiting for MR imaging would have delayed CEA or CAS.

The choice of CEA or CAS was determined by the surgeon or interventionist responsible for the patient. In general, CEA was considered as the first-line therapy for carotid stenosis with marked calcification and vascular kinking or tortuosity. CAS was indicated for patients with high bifurcations, for restenosis after CEA, and for those who preferred CAS to CEA. The physicians and patients talked about treatment policy and decided which methods should be performed on a patient-by-patient basis. In addition, the current study excluded patients with severe IHD or cardiac or pulmonary failure, which would be an impediment to general anesthesia. However, patients with mild IHD or cardiac failure or pulmonary failure, which did not impede general anesthesia, were included. Anesthesiologists were consulted about heart and pulmonary function and determined amenability to general anesthesia on a patient-by-patient basis. The local ethics committee approved this study, and informed consent was obtained from all subjects.

\section{MR Imaging Protocol}

MR imaging examinations were performed on an Achieva 3T system with Quasar Dual gradients (Philips Healthcare, Best, the Netherlands). After a baseline brain assessment including T1-weighted imaging, T2-weighted imaging, FLAIR, and DWI, all patients underwent MR imaging of the carotid arteries to determine the nature of the carotid plaque.

DWI was performed with a spin-echo-type EPI sequence: TR, $3000 \mathrm{~ms}$; TE, $48 \mathrm{~ms}$; FA, 90º ; sensitivity encoding, 3; FOV, $350 \mathrm{~mm}$ (rectangular FOV, 65\%); matrix, $142 \times 176$ (reconstruction $256 \times$ 256); section thickness, $4 \mathrm{~mm}$; gap, $1 \mathrm{~mm}$; sections, 24-26; fat suppression $=$ SPIR; $b$-value $=1000 \mathrm{~s} / \mathrm{mm}^{2} ; \mathrm{NEX}=2$; scanning time, 1 minute 3 seconds.

Black-blood images of the carotid arteries were acquired by using a double IR-TSE sequence and peripheral pulse gating with a 16channel neurovascular array coil. Parameters for the BB imaging se- quences were as follows: 1) T1WI-BB (2D double IR-TSE): TR, 1 cardiac R-R interval; TE, 9 ms; ETL, 6; FA, 50 FOV, $160 \times 160 \mathrm{~mm}$; matrix, $245 \times 256$ (reconstruction, $512 \times 512$ ); in-plane resolution, $0.65 \times 0.63 \mathrm{~mm}$ (reconstruction, $0.31 \times 0.31 \mathrm{~mm}$ ); section thickness, $3 \mathrm{~mm}$; sections, 15; scanning time, 3 minutes 30 seconds; 2) T2WI-BB (2D double IR-TSE); TR, 2 or 3 cardiac R-R intervals; TE, $40 \mathrm{~ms}$; ETL, 20; FA, $90^{\circ}$; FOV, $160 \times 160 \mathrm{~mm}$; matrix $=210 \times 256$ (reconstruction, $512 \times 512$ ); in-plane resolution, $0.76 \times 0.63 \mathrm{~mm}$ (reconstruction, $0.31 \times 0.31 \mathrm{~mm}$ ); section thickness, $3 \mathrm{~mm}$; sections, 15 ; scanning time, 5 minutes 30 seconds. The fat-suppression method was applied to suppress marked signal-intensity hyperintensity due to subcutaneous fat tissue by using a chemical shift selective fat-suppression technique of SPIR in T1WI-BB and T2WI-BB imaging. The median time between the preoperative MR imaging study and CEA or CAS was 11 days (range, 1-38 days). No new ischemic event occurred between the last MR imaging scan and the treatment in any enrolled patients.

\section{Image Analysis}

Plaque imaging data were transferred to a personal computer running open-source OsiriX imaging software (Version 3.5.1, http://www. osirix-viewer.com/) to assess the signal intensity of the carotid plaque. MR images were evaluated by 1 investigator (K.H.) blinded to the clinical characteristics and other imaging findings. The carotid bifurcation was used as a landmark for matching the 2 different contrastweighted images (T1WI-BB and T2WI-BB) at each section location. In each case, 10 locations ( $30 \mathrm{~mm}$ in length) centered at the narrowest lumen section were used for measurement of the T1-SIR of carotid plaque. We manually drew regions of interest around the identified plaque, including both hyperintense and hypointense regions. Then, we selected the highest value of the T1-SIR among the 10 sections for further analysis. The location of the region of interest was registered as within the main plaque (Fig 1). The OsiriX enabled us to coregister multisequence MR images and to copy and paste the same region of interest from T1WI-BB to T2WI-BB on the same location. Accordingly, the signal intensity at the corresponding location on the matched MR image was measured. The SIR of the plaque was then calculated relative to the adjacent sternocleidomastoid muscle as follows:

$$
\text { SIR }=\frac{\text { Signal Intensity of Plaque }}{\text { Signal Intensity of Sternocleidomastoid Muscle }} .
$$

We preliminarily determined interobserver variability of the SIR from 33 subjects in whom SIR was measured by 2 observers in randomly selected carotid cross-sections with the use of the Lin concordance correlation coefficient $(\rho)$. The $\rho$ was 0.97 and reflected high reproducibility. The Pearson correlation coefficient was 0.98 , which showed good precision. The location shift $(-0.14)$ and the scale shift (0.97) were small. These findings indicated good agreement.

\section{Therapeutic Procedures}

In cases of CEA, patients had been given at least 1 antiplatelet agent (aspirin, $100 \mathrm{mg}$ daily; cilostazol, $200 \mathrm{mg}$ daily; or clopidogrel, $75 \mathrm{mg}$ daily) for a minimum of 7 days before the procedure. CEA was performed with the patient under general anesthesia with somatosensory-evoked potential monitoring for selective placement of a shunt. In cases of CAS, patients had been given 2 antiplatelet agents (aspirin, $100 \mathrm{mg}$ daily, and ticlopidine, $200 \mathrm{mg}$ daily; cilostazol $200 \mathrm{mg}$ daily; or clopidogrel, $75 \mathrm{mg}$ daily) for a minimum of 7 days before the procedure. Stent procedures were performed with the patient under local 

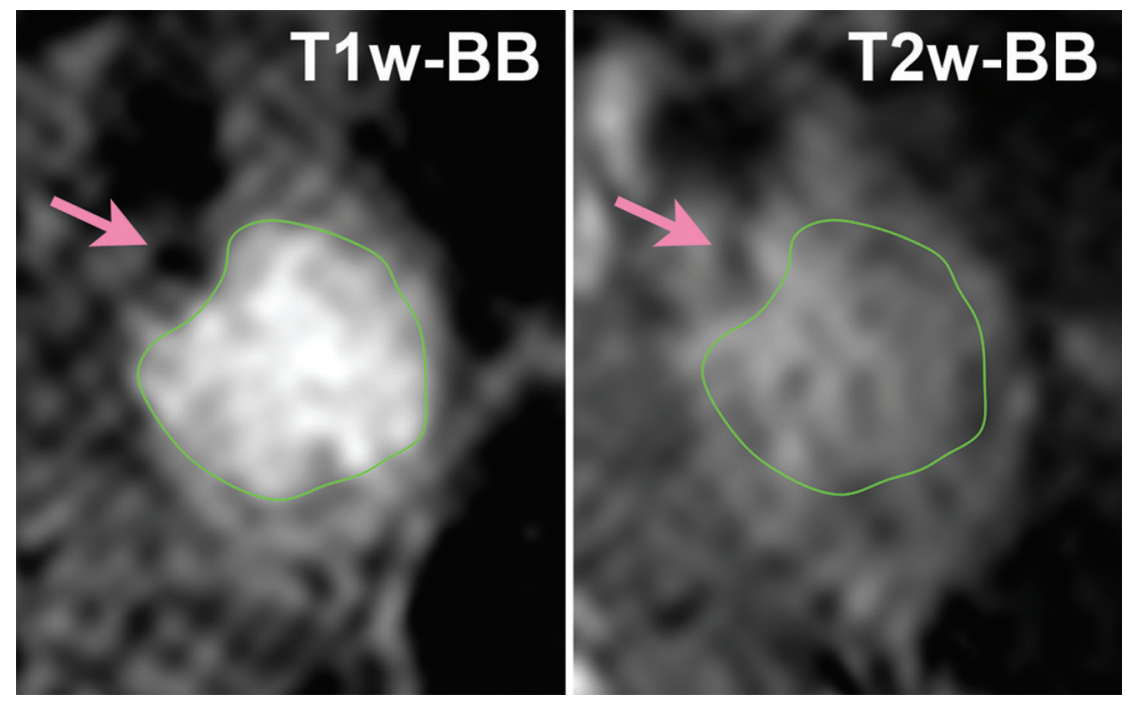

Fig 1. Coregistered multisequence MR imaging cross-sections of carotid plaque. Left: T1WI-BB. Right: T2WI-BB. The regions of interest are outlined in green. Arrows indicate the lumen of the ICA.

anesthesia via the percutaneous transfemoral route. An intravenous heparin bolus ( $5000 \mathrm{U}$ ) was given to elevate the activated clotting time between 2- and 2.5-fold above baseline values. Two different types of embolic protection devices were deployed in the ICA: PercuSurge Guardwire (Medtronic, Santa Rosa, California) $(n=20)$ and AngioGuard XP (Cordis, Miami, Florida) $(n=21)$. The vessel was predilated with a percutaneous transluminal angioplasty balloon catheter placed across the stenosis. Two types of stents were placed in the stenotic lesion: Precise (Cordis) $(n=39)$ or Wallstent (Boston Scientific, Natick, Massachusetts) $(n=2)$. Postdilation was performed with a 4.0 - or $5.0-\mathrm{mm}$ balloon catheter.

All patients underwent posttreatment MR imaging including DWI 1 day after treatment. The primary outcome was the occurrence of any new hyperintense DWI lesion on the posttreatment scan that had not been present on the pretreatment scan.

\section{Statistical Analysis}

Descriptive statistics are presented as mean \pm SD. A Welch 2-sample $t$ test was used to compare means. The Fisher exact test was used for proportion analysis.

A multivariate logistic regression model was applied to investigate the impact of various factors on the odds of new hyperintense lesions on posttreatment DWI. The predictor variables considered were age, sex, symptom, hypertension, DM, hyperlipidemia, statin administration, IHD, smoking, degree of ICA stenosis, T1-SIR, T2-SIR, and treatment (CEA or CAS). These 13 candidate predictors were chosen from the clinical literature and expert opinion. ${ }^{22}$ These candidate predictors were reduced to prevent overfitting. ${ }^{22,23}$ We focused mainly on the effect of T1-SIR, T2-SIR, degree of stenosis, and treatment (CEA or CAS). Regarding the other 9 predictors, we performed principal component analysis to reduce the dimensionality of the data. Consequently, these 9 predictors were reduced to a single variable by using the first-principal component of the principal component analysis, and this variable was designated as a clinical score. Then, the penalized maximum likelihood was used to accurately estimate the parameters of the models for model revision with additional shrinkage. ${ }^{23}$ Nonparametric CIs for parameter estimates were computed by using a bootstrap resampling procedure. ${ }^{23}$

The predictive accuracy of the full penalized logistic regression models was quantified by using the probability of concordance, the C-index, which is identical to area under receiver operating characteristic curve.

When the full penalized logistic regression was performed on the principal component, we used an ordinary linear model to decode internal regression coefficients to help understand the full model. Furthermore, we chose to approximate the model by using its most important predictors, by using a stepdown technique with ordinary least squares. ${ }^{23}$

Next, we diagrammed this model approximation so that all predicted values could be computed without the use of a computer. We drew a type of nomogram that converted each effect in the model to a $0-100$ scale, which was just proportional to the log odds. ${ }^{23}$ These points were added across predictors to derive the "total points," which were converted to predicted log odds and then to predicted probabilities. Consequently, a nomogram of the final approximate model was constructed to provide the overall probability of a specific outcome for an individual patient and to display the relationship between the different predictor variables and how this affects the outcomes. $^{23}$

Probability values of $P<.05$ were considered significant. Statisti$\mathrm{cal}$ analysis was performed with free open-source software (R2.12.2; R Foundation for Statistical Computing, the ade 4 package for principal component analysis, and the rms package for logistic regression and nomogram construction; http://www.r-project.org).

\section{Results}

During the time of the study, 104 patients (63 CEA and 41 CAS) fulfilled the criteria described above. Ninety-three patients were men and 11 were women. Mean age was $71.7 \pm 7.0$ years. The overall average of the degree of ICA stenosis was $75.6 \pm 10.2 \%$, according to the NASCET criteria. Fifty-three patients were symptomatic (34 CEA, 19 CAS), and 51 were asymptomatic (29 CEA, 22 CAS).

Major ipsilateral stroke occurred in 1 patient after CAS (2.4\%). No stroke occurred in patients after CEA. The difference in incidence of posttreatment stroke between CAS and CEA was not statistically significant. Three patients under- 


\begin{tabular}{|c|c|c|c|}
\hline \multirow[b]{2}{*}{ Predictor } & \multicolumn{2}{|c|}{$\begin{array}{c}\text { Hyperintense Lesions on } \\
\text { Postprocedural DWI }\end{array}$} & \multirow[b]{2}{*}{$P$ Value } \\
\hline & $\begin{array}{l}\text { Positive } \\
(n=25)\end{array}$ & $\begin{array}{c}\text { Negative } \\
(n=79)\end{array}$ & \\
\hline$\overline{\text { Age }(y r)}$ & $72.9 \pm 6.2$ & $71.3 \pm 7.2$ & .30 \\
\hline Male (\%) & $23(92 \%)$ & $70(89 \%)$ & 1 \\
\hline \multicolumn{4}{|l|}{ Vascular risk factors (\%) } \\
\hline Hypertension & $20(80 \%)$ & $54(68 \%)$ & .32 \\
\hline Hyperlipidemia & $21(84 \%)$ & $57(72 \%)$ & .30 \\
\hline Statin use & $20(80 \%)$ & $64(81 \%)$ & 1 \\
\hline DM & $10(40 \%)$ & $23(29 \%)$ & .33 \\
\hline Smoking & $17(68 \%)$ & $43(54 \%)$ & .26 \\
\hline IHD & $8(32 \%)$ & $16(20 \%)$ & .28 \\
\hline Symptomatic stenosis (\%) & $15(60 \%)$ & $38(48 \%)$ & .36 \\
\hline Clinical score & $0.073 \pm 0.97$ & $-0.023 \pm 1.02$ & .67 \\
\hline Degree of stenosis (\%) & $75.2 \pm 11.4$ & $75.8 \pm 9.8$ & .82 \\
\hline CAS $(\%)$ & $14(56 \%)$ & $27(34 \%)$ & .063 \\
\hline \multicolumn{4}{|l|}{ Plaque imaging } \\
\hline T1-SIR & $1.69 \pm 0.40$ & $1.36 \pm 0.29$ & .00054 \\
\hline T2-SIR & $1.49 \pm 0.45$ & $1.75 \pm 0.80$ & .045 \\
\hline
\end{tabular}

went CAS because of restenosis after previous CEA. None of them demonstrated new DWI lesions after CAS.

New hyperintense DWI lesions were recognized on the posttreatment scan in 25 patients (positive DWI group), and no new lesions in 79 patients (negative DWI group). Eleven (44\%) of 25 patients with new DWI lesions demonstrated new anatomically congruent lesions on T2-weighted or FLAIR images. Most of these lesions were subtle and only slightly hyperintense. One hyperintense DWI lesion was recognized in 16 patients and $\geq 2$ lesions in the other 9 patients. One patient treated by CAS and 1 treated by CEA had contralateral hyperintense DWI lesions in addition to ipsilateral lesions. The average number of new hyperintense DWI lesions was 3.1, ranging from 1 to 17 (median, 1).

\section{Univariate Analysis}

In univariate analysis, demographic and clinical characteristics did not differ significantly between negative and positive DWI groups (Table 1). Incidence of new hyperintense DWI lesions was higher in the CAS group $(14 / 41,34 \%)$ than in the CEA group (11/63;17\%), though the difference was just under statistical significance $(P=.063)$ (Table 1$)$. The quality of plaque imaging was sufficient for measurement of SIR in all patients. MR plaque imaging demonstrated a significantly higher value of T1-SIR $(P=.00054)$ and a significantly lower value of T2-SIR $(P=.045)$ in the positive DWI group than in the negative DWI group (Table 1 ).

We also found that T1-SIR was significantly higher in the symptomatic group than in the asymptomatic group (1.53 versus $1.34, P=.0058$ ) and that T2-SIR was significantly lower in the symptomatic group than in the asymptomatic group (1.52 versus $1.86, P=.019)$.

Seven $(11 \%)$ of 63 patients with CEA received an intraoperative shunt. No significant difference in the incidence of new DWI lesions after surgery was found between CEAs with shunt (1/7) and CEAs without shunt (10/56). Only 2 patients received a shunt because of reduction of the amplitude of the somatosensory-evoked potential. These 2 patients demon- strated no new DWI lesions after CEA. The other 5 patients received a shunt because the somatosensory-evoked potential could not be measured for some reason.

\section{Multivariate Analysis and Construction of a Preliminary Predictive Model and Nomogram}

The ORs and 95\% CIs for the association of the predictors with the risk of new hyperintense lesions on posttreatment DWI in the full penalized logistic model are presented in Fig 2. T1-SIR (OR, 3.99; 95\% CI, 2.18-7.31; $P<.0001$, estimated odds of outcome for a 0.5 increase in T1-SIR) and CAS (OR, 2.06; $95 \%$ CI, $1.01-4.24 ; P=.048$ compared with CEA) were significantly associated with increased odds of new ischemic lesions on posttreatment DWI. On the other hand, T2-SIR (OR, 0.74; $95 \%$ CI, $0.55-0.98 ; P=.037$, estimated odds of outcome for a 0.5 increase in T2-SIR) was significantly associated with decreased odds of new ischemic lesions on posttreatment DWI. T1-SIR was the most important predictor. The degree of stenosis and clinical score were not found to be statistically significant. The discriminative value of this full penalized logistic regression model, as expressed by the C-index, was 0.79 (95\% CI, 0.69-0.89), which indicated some utility in predicting the response of individual patients.

Effects of all 13 predictors in the ordinary linear model to decode internal regression coefficients of the full penalized logistic model are shown on a common scale in Fig 3. It is obvious that 4 predictors (age, symptom status, hypertension, and IHD) have almost no effect and 5 predictors (sex, DM, smoking, hyperlipidemia, and statin use) have little effect on the appearance of new DWI lesions after treatment in this model. The stepdown technique with ordinary least squares removed these 9 variables and constructed an approximate predictive model that had $R^{2}$ against the full penalized logistic model of $>0.99$. This approximate preliminary predictive model is presented as a nomogram in Fig 4. Four axes show effects of the 4 predictors plus reading. Figure 5 and Table 2 give an example by using the nomogram. A 68-year-old man gets 2 points for having a right carotid stenosis of $85 \%, 30$ points for having a T1-SIR of 1.4, forty-nine points for having a T2-SIR of 1.1, and 0 points for CEA or 14 points for CAS; that man gets 81 total points (for CEA) or 95 points (for CAS), respectively. This corresponds to a probability of a new DWI lesion of $19 \%$ or $34 \%$, respectively.

\section{Discussion}

Our data demonstrated that higher T1-SIR and lower T2-SIR and CAS were significantly associated with new ischemic lesions on posttreatment DWI. T1-SIR was the most important predictor. The value of the C-index of the current preliminary model indicated some utility in prediction. Patients with carotid stenosis have many combinations of clinical predictors that can be independently measured and then analyzed together to predict a specific outcome. This individual specific outcome of new DWI lesions after treatment can now be estimated through the use of the preliminary prediction model and nomogram developed in the current study.

The current results provide evidence that carotid plaque demonstrating both higher T1-SIR and lower T2-SIR, which suggests acute intraplaque hemorrhage, can identify patients with active thromboembolic plaque causing posttreatment 


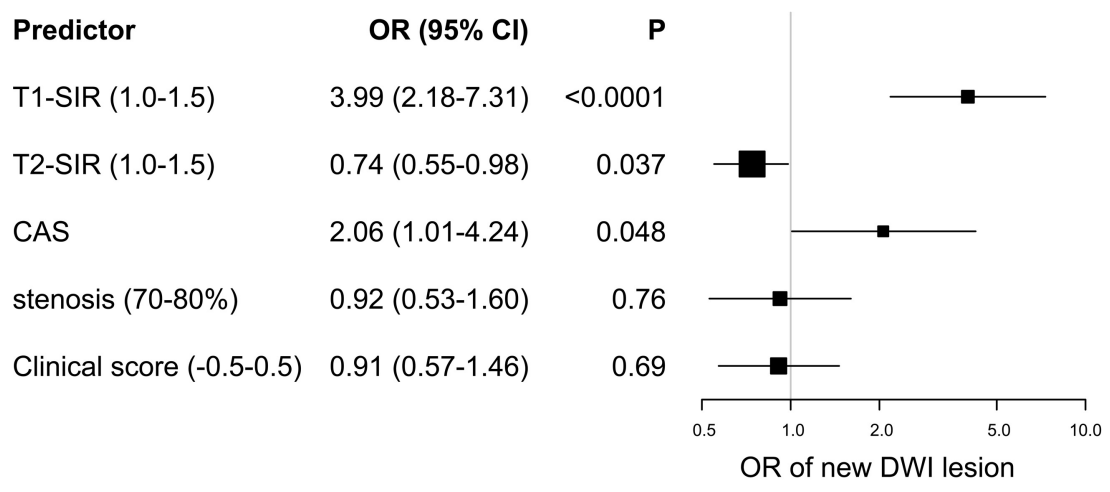

Fig 2. Forest plot of ORs of the full penalized logistic regression model for new occurrences of hyperintense lesions on DWI after CEA or CAS. All odds of continuous variables (T1-SIR, T2-SIR, stenosis, and clinical score) are represented as odds per increase, described in parentheses. Squares and horizontal bars represent ORs and $95 \%$ Cls, respectively, on a log scale. The size of the squares represents precision.

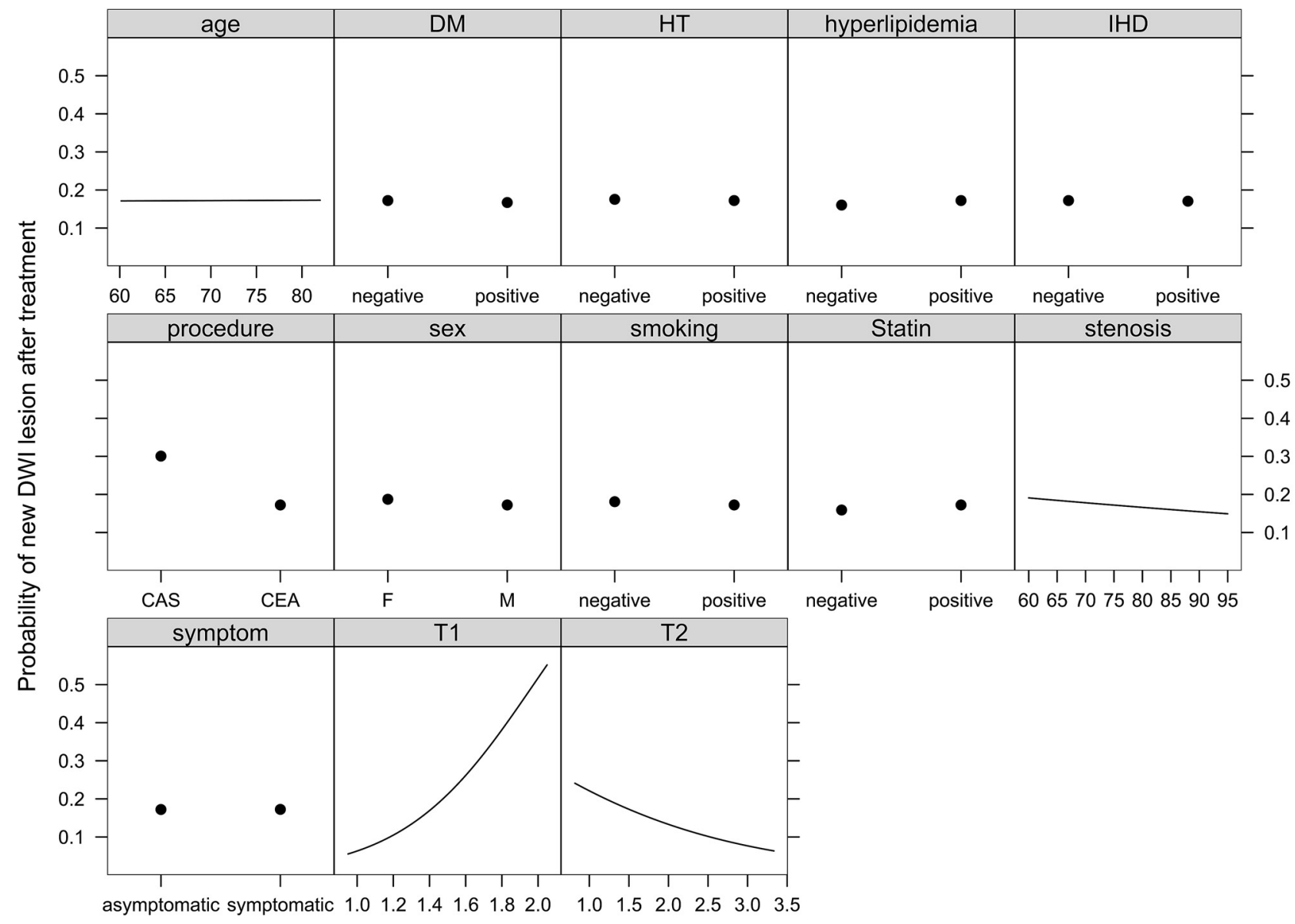

Fig 3. Effects of all of the predictors on the probability of new occurrences of hyperintense lesions on DWI after CEA or CAS, holding other predictors to their medians. See text for details.

ischemic lesions in the brain. Intraplaque hemorrhage has been thought to be a critical factor in atherosclerotic plaque growth and destabilization because erythrocyte membranes within atherosclerotic plaque are capable of providing a substantial amount of lipid to the necrotic core and promoting excessive macrophage infiltration. ${ }^{24,25}$ The largest histologic study of symptomatic carotid plaques demonstrated that intraplaque hemorrhage was independently associated with cap rupture. ${ }^{6}$ Recent studies with carotid MR imaging demonstrated that intraplaque hemorrhage was associated with an increased risk of subsequent ischemic events on clinical fol- low-up ${ }^{16,17}$ and embolization during CEA. ${ }^{19}$ In these studies, intraplaque hemorrhage was defined as hyperintense signal intensity compared with adjacent muscle on T1-weighted BB MR imaging. The latest single-center prospective study demonstrated that CAS, symptomatic stenosis, and T1-SIR were significant predictors of new hyperintense lesions after treatment. ${ }^{21}$ These results are consistent with the current results.

Our preliminary prediction model also demonstrated the negative effect of T2-SIR on the postoperative occurrence of new ischemic lesions. Previous studies demonstrated that acute intraplaque hemorrhage was histologically character- 
Points

stenosis

T1

$\mathrm{T} 2$

procedure

Total Points

\section{$\begin{array}{ccc}11 & & \\ 95 & 70 & 50\end{array}$}

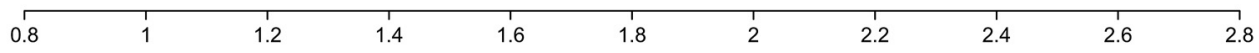

\begin{tabular}{llllllllllll}
\hline 5.5 & 5 & 4.5 & 4 & 3.5 & 3 & 2.5 & 2 & 1.5 & 1 & 0.5 & 0 \\
& \multicolumn{2}{c|}{ CAS }
\end{tabular}

CEA

020

40

60

80

100

120

140

160

180

Probability of new HI on postop. DWI

$\begin{array}{llllllllllll}0.05 & 0.1 & & 0.2 & 0.3 & 0.4 & 0.5 & 0.6 & 0.7 & 0.8 & 0.9 & 0.95\end{array}$

Fig 4. Preliminary nomogram for predicting the probability of new occurrences of hyperintense lesions on DWI after treatment (CEA or CAS) from the full penalized logistic model, by using an approximate model fitted by using ordinary least squares $\left(R^{2}=0.992\right.$ against the predicted logits of the full penalized logistic model). Points are assigned for each of the 4 predictors. The total points correspond to an absolute predicted risk for occurrence of new hyperintense lesions on DWI after treatment. For each predictor, read the points assigned on the $0-100$ scale and add these points. Read the results on the Total Points scale and then read the corresponding predictions below it. Postop. indicates postoperative.
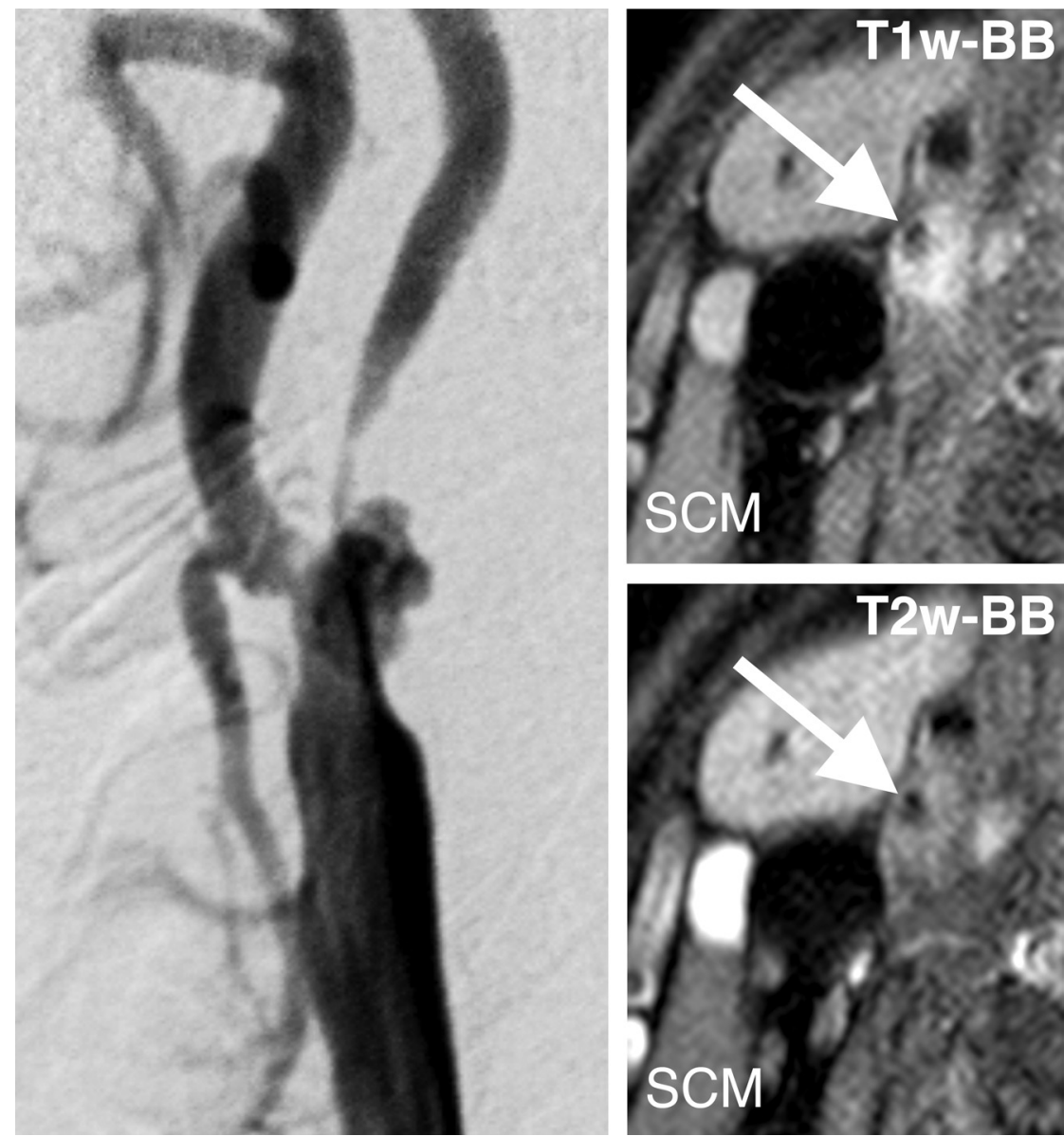

Fig 5. An example using the preliminary nomogram for predicting the probability of new occurrences of hyperintense lesions on DWI after treatment (CEA or CAS). Left: right carotid angiogram. Right upper: T1WI-BB. Right lower: T2WI-BB. Arrows indicate the lumen of the ICA. See text and Table 2 for details.

ized by intact red blood cells with intracellular methemoglobin and appeared hyperintense on T1-weighted images and iso- to hypointense on T2-weighted images. ${ }^{10,18}$ Recent hemorrhage was histologically characterized by lytic red blood cells with extracellular methemoglobin and appeared hyperintense on all sequence images. Acute hemorrhage was reported to be significantly associated with ischemic symptoms. ${ }^{18}$ Therefore, the negative effect of T2-SIR seems reasonable. Accordingly, measurement of both T1-SIR and T2-SIR is useful for the detection of acute intraplaque hemorrhage, which is most risky for embolic ischemia.

We focused on SIR from multisequence MR imaging. Further quantification of other features such as lipid-rich necrotic core and ruptured fibrous cap might provide a more accurate 


\begin{tabular}{|c|c|c|c|c|}
\hline \multirow[b]{3}{*}{ Predictor } & \multicolumn{4}{|c|}{ Example } \\
\hline & \multicolumn{2}{|c|}{ Risk of CEA } & \multicolumn{2}{|c|}{ Risk of CAS } \\
\hline & Values & Points & Values & Points \\
\hline Stenosis (\%) & 85 & 2 & 85 & 2 \\
\hline T1-SIR & 1.4 & 30 & 1.4 & 30 \\
\hline T2-SIR & 1.1 & 49 & 1.1 & 49 \\
\hline CEA or CAS & CEA & 0 & CAS & 14 \\
\hline Total points & \multirow{2}{*}{\multicolumn{2}{|c|}{$\begin{array}{c}81 \\
19 \%\end{array}$}} & \multirow{2}{*}{\multicolumn{2}{|c|}{$\begin{array}{c}95 \\
34 \%\end{array}$}} \\
\hline $\begin{array}{c}\text { Probability of new HI } \\
\text { on postop. DWI }\end{array}$ & & & & \\
\hline
\end{tabular}

Note:- postop. indicates postoperative; HI, hyperintensity.

prediction of the occurrence of new DWI lesions after treatment though such a subanalysis would not be reliable because of the insufficient statistical power due to the small number of patients in the current study. In clinical practice, however, quantification of SIR was easy and would be sufficient to detect intraplaque hemorrhage.

An increase in signal-to-noise ratio and spatial resolution is commonly considered 1 of the major advantages of high-field imaging, such as the $3 \mathrm{~T} \mathrm{MR}$ imaging we used. At higher field strengths, however, the increased susceptibility of calcification and paramagnetic ferric iron in hemorrhage may alter quantification and/or detection. Nevertheless, imaging criteria at $1.5 \mathrm{~T}$ for carotid plaque interpretation were reported to be applicable at $3 \mathrm{~T}^{26}$

One systematic review demonstrated that new DWI lesions occur more frequently after CAS (44\%) than after CEA (11\%) (OR, 6.1; 95\% CI, 4.19-8.87). ${ }^{4}$ Another meta-analysis of nonrandomized studies showed that the aggregate OR of new ischemic lesions after treatment was 6.71 (95\% CI, 4.57-9.87) favoring CEA (CEA, 10\%; CAS, 42\%). ${ }^{27}$ Furthermore, the most recent prospective randomized study demonstrated that $50 \%$ of the CAS group and 17\% of the CEA group had new DWI lesion detected on posttreatment scans (OR, 5.21; 95\% CI, $2.78-9.79) .{ }^{27}$ The incidence and OR of CAS for DWI lesions in the current study demonstrated a similar trend though they were smaller than those in previous studies. These coincidences argue against a selection bias - that is, high cardiovascular risk profiles were assigned to the CAS group in the current study.

The degree of stenosis had no significant effect on the occurrence of postoperative new ischemic lesions, which corresponds to previous clinical trials indicating no significant effect of the degree of stenosis on the perioperative stroke rate. ${ }^{1,2}$ Nine clinical factors also demonstrated no significant effect. Even symptom status did not have a significant effect on postoperative new ischemic lesions, though symptomatic carotid stenosis demonstrated significantly higher T1-SIR and significantly lower T2-SIR than asymptomatic stenosis in the univariate analysis. A larger number of patients in a future study may be able to provide a more sensitive estimation of the effect of each clinical factor because of sufficient statistical power.

Only 1 clinical stroke occurred, and all of the other DWI lesions were subclinical in the current study. The clinical impact of these "silent" ischemic lesions has not been fully clarified. Slight neuropsychological deterioration such as cognitive decline or memory disturbance and personality changes can be easily missed on routine examination because specific neuropsychological tests are required for diagnosis. However, there is increasing evidence that the cumulative burden of ischemic lesions causes neuropsychological deficits. ${ }^{28,29}$ The latest randomized study demonstrated that CAS had a small but statistically significant detrimental effect on cognitive functioning at 6 months after treatment for symptomatic carotid stenosis, an effect not observed with CEA, probably because of a more than 2-fold higher rate of new ischemic lesions on DWI after CAS than after CEA. ${ }^{30}$ Accordingly, considering the low stroke and death rates of CEA and CAS in recent clinical trials ${ }^{31,32}$ and the current study, even subclinical infarction might be a factor to be reckoned with for treatment strategy. Our preliminary predictive model could provide some information about the probability of new ischemic lesions on DWI after treatment. For example, if a patient is in stable condition and the probability of a new DWI lesion after treatment is below $50 \%$, the patient could select a treatment option according to personal preference. If the probability of new DWI lesion after treatment with 1 treatment option (CEA or CAS) exceeds $50 \%$, another option might be recommended.

There are some limitations to the current study. First, carotid plaque MR imaging might preferably be incorporated into a randomized trial comparing CEA with CAS. In the current study, CEA and CAS were not randomly allocated to patients. However, patient clinical characteristics did not differ significantly between the CEA and CAS groups in the current study. Furthermore, we analyzed the data with multivariate logistic regression, which allows control of confounding variables. Therefore, it seems reasonable to state that higher T1SIR and lower T2-SIR and CAS are significant risk factors for posttreatment DWI ischemic lesions after controlling for other variables. Second, a recent study reported that MPRAGE, compared with the fast spin-echo sequences, demonstrated a higher diagnostic capability for the detection and quantification of intraplaque hemorrhage. ${ }^{12}$ The initial protocol of the MR imaging sequences in the current study did not include an MPRAGE sequence. We regarded consistency of data as important and kept using double IR-TSE in the current study. Third, the small sample size and thus the imprecision of any predictive values coming from the model are a main limitation of our study. Accordingly, we did not include other important risk factors such as plaque volume and ulceration ${ }^{33}$ into predictors because the addition of these predictors would have caused further overfitting due to the small number of events; thus, we call our prediction model preliminary. Further collection of patients and validation of the current preliminary model are needed in a future study.

\section{Conclusions}

We found that higher T1-SIR and lower T2-SIR and CAS were significantly associated with the occurrence of new DWI lesions after CEA or CAS. T1-SIR was the most important predictor. The individual outcome can be predicted by the use of the nomogram we developed in the current study. Our preliminary prediction model and nomogram may provide information about the probability of posttreatment ischemic lesions and assist clinicians and patients in their decisionmaking regarding treatment strategies for carotid stenosis. 


\section{Acknowledgments}

The authors express their gratitude to Hideaki Kawamitsu and Nobukazu Aoyama for technical assistance in MR imaging study.

Disclosures: Eiji Kohmura—UNRELATED: Consultancy. Medtronic, Comments: surgical drill, Grants/Grants Pending: Ministry of Education, Culture, Sports, Science, and Technology in Japan, ${ }^{*}$ Payment for Lectures (including service on Speakers Bureaus): Novartis, Astellas, Nakamura Memorial Hospital, Daiichi Sankyo, Comments: brain tumor surgery, Royalties: Muranaka Co Ltd, Comments: surgical instruments. * Money paid to the institution.

\section{References}

1. Rothwell PM, Eliasziw M, Gutnikov SA, et al. Analysis of pooled data from the randomised controlled trials of endarterectomy for symptomatic carotid stenosis. Lancet 2003;361:107-16

2. Executive Committee for the Asymptomatic Carotid Atherosclerosis Study. Endarterectomy for asymptomatic carotid artery stenosis: Executive Committee for the Asymptomatic Carotid Atherosclerosis Study. JAMA 1995;273:1421-28

3. Yadav JS, Wholey MH, Kuntz RE, et al. Protected carotid-artery stenting versus endarterectomy in high-risk patients. N Engl J Med 2004;351:1493-501

4. Schnaudigel S, Groschel K, Pilgram SM, et al. New brain lesions after carotid stenting versus carotid endarterectomy: a systematic review of the literature. Stroke 2008;39:1911-19

5. Spagnoli LG, Mauriello A, Sangiorgi G, et al. Extracranial thrombotically active carotid plaque as a risk factor for ischemic stroke. JAMA 2004;292:1845-52

6. Redgrave JN, Lovett JK, Gallagher PJ, et al. Histological assessment of $\mathbf{5 2 6}$ symptomatic carotid plaques in relation to the nature and timing of ischemic symptoms: the Oxford plaque study. Circulation 2006;113:2320-28

7. Saam T, Ferguson MS, Yarnykh VL, et al. Quantitative evaluation of carotid plaque composition by in vivo MRI. Arterioscler Thromb Vasc Biol 2005;25: 234-39

8. Cai J, Hatsukami TS, Ferguson MS, et al. In vivo quantitative measurement of intact fibrous cap and lipid-rich necrotic core size in atherosclerotic carotid plaque: comparison of high-resolution, contrast-enhanced magnetic resonance imaging and histology. Circulation 2005;112:3437-44

9. Yuan C, Mitsumori LM, Ferguson MS, et al. In vivo accuracy of multispectral magnetic resonance imaging for identifying lipid-rich necrotic cores and intraplaque hemorrhage in advanced human carotid plaques. Circulation 2001; 104:2051-56

10. Chu B, Kampschulte A, Ferguson MS, et al. Hemorrhage in the atherosclerotic carotid plaque: a high-resolution MRI study. Stroke 2004;35:1079-84

11. Moody AR, Murphy RE, Morgan PS, et al. Characterization of complicated carotid plaque with magnetic resonance direct thrombus imaging in patients with cerebral ischemia. Circulation 2003;107:3047-52

12. Ota H, Yarnykh VL, Ferguson MS, et al. Carotid intraplaque hemorrhage imaging at 3.0-T MR imaging: comparison of the diagnostic performance of three T1-weighted sequences. Radiology 2010;254:551-63

13. Hatsukami TS, Ross R, Polissar NL, et al. Visualization of fibrous cap thickness and rupture in human atherosclerotic carotid plaque in vivo with high-resolution magnetic resonance imaging. Circulation 2000;102:959-64

14. Bitar R, Moody AR, Leung G, et al. In vivo 3D high-spatial-resolution MR imaging of intraplaque hemorrhage. Radiology 2008;249:259-67
15. Yoshida K, Narumi O, Chin M, et al. Characterization of carotid atherosclerosis and detection of soft plaque with use of black-blood MR imaging. AJNR Am J Neuroradiol 2008;29:868-74

16. Takaya N, Yuan C, Chu B, et al. Association between carotid plaque characteristics and subsequent ischemic cerebrovascular events: a prospective assessment with MRI-initial results. Stroke 2006;37:818-23

17. Altaf N, MacSweeney ST, Gladman J, et al. Carotid intraplaque hemorrhage predicts recurrent symptoms in patients with high-grade carotid stenosis. Stroke 2007;38:1633-35

18. Saam T, Cai J, Ma L, et al. Comparison of symptomatic and asymptomatic atherosclerotic carotid plaque features with in vivo MR imaging. Radiology 2006;240:464-72

19. Altaf N, Beech A, Goode SD, et al. Carotid intraplaque hemorrhage detected by magnetic resonance imaging predicts embolization during carotid endarterectomy. J Vasc Surg 2007;46:31-36

20. Yamada K, Kawasaki M, Yoshimura S, et al. Prediction of silent ischemic lesions after carotid artery stenting using integrated backscatter ultrasound and magnetic resonance imaging. Atherosclerosis 2010;208:161-66

21. Yamada K, Yoshimura S, Kawasaki M, et al. Embolic complications after carotid artery stenting or carotid endarterectomy are associated with tissue characteristics of carotid plaques evaluated by magnetic resonance imaging. Atherosclerosis 2011;215:2:399-404

22. Steyerberg EW. Clinical Prediction Models: A Practical Approach to Development, Validation, and Updating. New York: Springer-Verlag; 2009:1-507

23. Harrell FE Jr. Regression Modeling Strategies: With Applications to Linear Models, Logistic Regression, and Survival Analysis. New York: Springer-Verlag; 2001:1568

24. Kolodgie FD, Gold HK, Burke AP, et al. Intraplaque hemorrhage and progression of coronary atheroma. N Engl J Med 2003;349:2316-25

25. Virmani R, Kolodgie FD, Burke AP, et al. Atherosclerotic plaque progression and vulnerability to rupture: angiogenesis as a source of intraplaque hemorrhage. Arterioscler Thromb Vasc Biol 2005;25:2054-61

26. Underhill HR, Yarnykh VL, Hatsukami TS, et al. Carotid plaque morphology and composition: initial comparison between 1.5- and 3.0-T magnetic field strengths. Radiology 2008;248:550-60

27. Bonati LH, Jongen LM, Haller S, et al. New ischaemic brain lesions on MRI after stenting or endarterectomy for symptomatic carotid stenosis: a substudy of the International Carotid Stenting Study (ICSS). Lancet Neurol 2010;9: 353-62

28. Bendszus M, Stoll G. Silent cerebral ischaemia: hidden fingerprints of invasive medical procedures. Lancet Neurol 2006;5:364-72

29. Leary MC, Caplan LR. Technology insight: brain MRI and cardiac surgerydetection of postoperative brain ischemia. Nat Clin Pract Cardiovasc Med 2007; 4:379-88

30. Altinbas A, van Zandvoort MJ, van den Berg E, et al. Cognition after carotid endarterectomy or stenting: A randomized comparison. Neurology 2011;77: 1084-90. Epub 2011 Aug 31

31. Brott TG, Hobson RW, Howard G, et al. Stenting versus endarterectomy for treatment of carotid-artery stenosis. N Engl J Med 2010;363:1 1-23

32. Bonati LH, Dobson J, Algra A, et al for the Carotid Stenting Trialists' Collaboration. Short-term outcome after stenting versus endarterectomy for symptomatic carotid stenosis: a preplanned meta-analysis of individual patient data. Lancet 2010;376:1062-73, Epub 2010 Sep 15

33. U-King-Im JM, Fox AJ, Aviv RI, et al. Characterization of carotid plaque hemorrhage: a CT angiography and MR intraplaque hemorrhage study. Stroke 2010;41:1623-29 